\title{
«Bulletin de la Société des Amis de Rober Challe»,
}

n. 10

\section{Franco Piva}

\section{(e) OpenEdition \\ 1 Journals}

\section{Edizione digitale}

URL: http://journals.openedition.org/studifrancesi/33247

DOI: $10.4000 /$ studifrancesi.33247

ISSN: 2421-5856

\section{Editore}

Rosenberg \& Sellier

\section{Edizione cartacea}

Data di pubblicazione: 1 décembre 2005

Paginazione: 644-645

ISSN: 0039-2944

\section{Notizia bibliografica digitale}

Franco Piva, ««Bulletin de la Société des Amis de Rober Challe», n. 10», Studi Francesi [Online], 147 (XLX | III) | 2005, online dal 01 novembre 2015, consultato il 18 avril 2021. URL: http:// journals.openedition.org/studifrancesi/33247 ; DOI: https://doi.org/10.4000/studifrancesi.33247

Questo documento è stato generato automaticamente il 18 avril 2021.

\section{(c) (i) (9)}

Studi Francesi è distribuita con Licenza Creative Commons Attribuzione - Non commerciale - Non opere derivate 4.0 Internazionale. 


\title{
«Bulletin de la Société des Amis de Rober Challe», n. 10
}

\author{
Franco Piva
}

\section{NOTIZIA}

«Bulletin de la Société des Amis de Rober Challe», n. 10 (2004), pp. 72.

1 Questo numero del «Bulletin», curato cone sempre da Jacques Cormier, comprende la quinta ed ultima delle histoires che apparvero sotto il titolo delle Illustres Françaises dal 1713 al 1780. Si tratta dell'Histoire de Monsieur de Bréville et de Mademoiselle de Beaumont. Inserita nella raccolta di Challe nell'edizione del 1723, essa costituisce quindi la seconda "continuation», dopo che nell'edizione dell'anno precedente era apparsa l'Histoire de Vallebois et de Charlotte de Pontais, e prima che due anni più tardi vi fossero pubblicate l'Histoire de M. de Livry et de Mademoiselle de Mancigni e l'Histoire de M. de Salvagne et de Mme de Villiers. Riprodotta identica nelle due edizioni successive delle Illustres Françaises, nel 1725 l'Histoire fu spostata «et quelque peu retouchée [come fa osservare e dimostra Jacques Cormier] en vue de mieux s'insérer dans le corpus des Illustres Françaises». A differenza delle altre novelle apocrife, l'Histoire de Monsieur de Bréville non figura in tutte le edizioni successive delle Illustres Françaises ma solo in quelle che derivano dall'edizione (G) del 1725.

2 Al pari delle altre histoires apocrife, neppure l'Histoire de Monsieur de Bréville et de Mademoiselle de Beaumont s'inserisce molto bene nel cadre delle Illustrtes Françaises: non sfrutta alcuna delle pierres d'attente che Challe vi aveva inserito, né è annunciata da qualcuno degli interventi di Des Ronais, di Dupuis o di Contamine. Richiama per altro verso abbastanza da vicino l'Histoire de Jussy et de Babet Fenouil, alla quale si ispira apertamente anche se si sviluppa in maniera abbastanza originale, con l'introduzione, per esempio, di due personaggi malfaisants, quello della madre e del fratello cupido, che nella histoire svolgono un ruolo non trascurabile. Il personaggio più interessante è certamente quello di Mlle de Beaumont che unisce la duplicità di Manon Dupuis allo 
spirito d'iniziativa di Babet Fenouil; peccato che delle due eroine challiane non possieda la coerenza psicologica. Come ha fatto notare Michèle Weil, "[l'histoire de Mademoiselle de Beaumont] et sa conduite ressemblent fort à celles de Mademoiselle Fenouil, au point de sembler une pastiche plutôt qu'une continuation»; tuttavia «les dénouements diffèrent comme les portraits: Mademoiselle de Beaumont devient inexplicablement infidèle, à la fin de l'histoire, perfide, incompréhensible tant pour les personnages que pour l'auteur-narrateur et le lecteur, alors que Babet Fenouil reste fidèle à son amant pendant ses sept ans d'exil et que les héroines dites infidèles, Manon et Silvie, sont réhabilitées». Di modo che «Physiquement et moralement, Mademoiselle de Beaumont ne peut pas être une «Illustre Française». Un giudizio severo, ancorché giusto, il quale tuttavia non può, né deve distogliere il lettore di Challe dal considerare con una certa attenzione anche questa «continuation». 